\title{
DIFERENCIAS EN LOS RENDIMIENTOS DE LOS PROCESOS CONSTRUCTIVOS DE PLACA ENTREPISO Y MUROS DE CARGA MEDIANTE EL SISTEMA DE CONSTRUCCIÓN INDUSTRIALIZADO
}

\section{DIFFERENCES IN THE PERFORMANCE OF THE CONSTRUCTION PROCESSES OF FLOOR SLABS AND LOAD-BEARING WALLS USING THE INDUSTRIALIZED CONSTRUCTION SYSTEM}

\section{Javier Alfonso Cárdenas Gutiérrez \\ José Leonardo Jácome Carrascal² \\ Mawency Vergel Ortega ${ }^{3}$}

Grupo de investigación en transporte y obras civiles - GITOC

\footnotetext{
$1 \quad$ Magíster en Administración de Empresas con Especialidad en Dirección de Proyectos. Universidad Francisco de Paula Santander Cúcuta - Colombia, Orcid: 00000002-9894-0177 grupo de investigación en transporte y obras civiles - GITOC

Email: javieralfonsocg@ufps.edu.co

2 Magister en Ingeniería civil. Maestrante en educación matemática. Universidad Francisco de Paula Santander Cúcuta - Colombia, Orcid:

Email: jose.jacome@ufps.edu.co Grupo de investigación en estadística Graunt

3 Doctora en Educación. Postdoctora en Imaginarios y representaciones sociales. Docente y Directora del Departamento de Matemáticas y Estadística. Filiación: Universidad Francisco de Paula Santander. Correo electrónico: mawency@ufps.edu.co. Orcid: https://orcid.org/0000-00018285-2968
}

\section{RESUMEN}

En la historia de la humanidad se evidencia el constate desarrollo y evolución en todos los aspectos necesarios que permiten garantizar nuestra supervivencia, siendo la construcción uno de ellos. A medida que el ser humano se vuelve consciente y adquiere el conocimiento necesario, obtiene la capacidad para poder salvaguardar su bienestar y el de quienes lo rodean, mediante la invención de diferentes tipos 
de infraestructuras utilizando inteligentemente los materiales que tiene a su disposición.

Por lo tanto, gracias al avance de la tecnología que hoy en día tenemos y la creencia en la mejora continua, en la industria de la construcción existen y se utilizan diferentes métodos constructivos según las necesidades del proyecto. Para este caso de estudio se evaluará la construcción de las placas de entre piso y muros de carga en viviendas unifamiliares con el fin de determinar y analizar los rendimientos obtenidos en estos dos elementos estructurales.

\section{PALABRAS CLAVE:}

Sistemas constructivos, industria de la construcción, rendimientos, elementos estructurales, vivienda unifamiliar.

\section{ABSTRACT}

The history of mankind shows the constant development and evolution in all the necessary aspects that allow us to guarantee our survival, construction being one of them. As the human being becomes conscious and acquires the necessary knowledge, he obtains the capacity to be able to safeguard his well-being and that of those around him, through the invention of different types of infrastructures using intelligently the materials at his disposal.

Therefore, thanks to the advancement of technology that we have today and the belief in continuous improvement, different construction methods exist and are used in the construction industry according to the needs of the project. For this case study we will evaluate the construction of floor slabs and load-bearing walls in singlefamily houses in order to determine and analyse the performance obtained in these two structural elements.

\section{KEY WORDS:}

Building systems, construction industry, performance, structural elements, single-family housing.

\section{INTRODUCCIÓN}

Los sistemas constructivos se desarrollaron con el fin de entregar una opción eficiente y segura a las personas que han decidido construir cualquier tipo de infraestructura. De tal forma, estos sistemas se encuentran conformados por diferentes tipos de elementos que se relacionan entre sí, manteniendo un vínculo interno que de forma segura conserva la estabilidad de la construcción realizada. Sin embargo, esto variará mucho según la manera en que los diferentes elementos se encuentren organizados, así mismo como dependerán de la forma en la que se construyan. (Espinoza \& Morán, 2017)

En la actualidad, la construcción se encuentra en un momento de auge e implementación de nuevas tecnologías, permitiendo la innovación de los diferentes procesos constructivos que mantenían un ritmo de trabajo lento y convencional en cada una de sus actividades (Aguirre Almache \& Zúñiga Chimbo, 2019).

Sin embargo, la innovación de estos sistemas no puede ser vista únicamente desde la conveniencia de la región o del contexto, sino que necesitan abordarse en todos los ámbitos que los constituyen, con el fin de establecer métodos aplicados tanto a la habitabilidad, así como a la sustentabilidad y los diferentes impactos que tienen. $Y$ finalmente establecer una correcta toma de decisiones, determinar si se deben proponer nuevos mecanismos 0 mejorar los existentes teniendo como premisa el establecimiento de un entorno de bienestar y por ende una mejor calidad de vida (Flores, 2018).

Uno de los sistemas innovadores actualmente, es el sistema industrializado, 
el cual permite construir de manera rápida y eficiente, siendo muy atractivo para las empresas constructoras debido a que posee procedimientos que facilitan la realización de viviendas unifamiliares en serie, y a su vez cumple con las normas establecidas (Tovar, 2021), teniendo como objetivo principal el aumento de la productividad y rendimiento, siendo este ultimo el gasto de materiales y mano de obra en un determinado tiempo (CárdenasGutiérrez et al., 2020).

Así mismo, esta alternativa constructiva termina siendo una solución a las diferentes problemáticas sociales encontradas en los diferentes países latinoamericanos que han presentando movimientos migratorios masivos de sus zonas rurales hacia los centros poblados en busca de mejores oportunidades desde hace algunas décadas (Orozco Herrera \& López Toro, 2020). De tal forma, los gobiernos ven como una gran oportunidad esta metodología constructiva e invierten en ella con la entrega de viviendas unifamiliares subsidiadas y así, poder satisfacer la alta demanda de familias que carecen del calor de un hogar debido a la escasez de recursos, problemáticas de orden público o calamidades domésticas (Mosquera Ayala \& Noreña Trejos, 2021; Puerto Castro, 2015) .

Por lo tanto, la implementación y sobre todo la filosofía de la mejora continua con respecto no solo a este sistema constructivo, sino todos los que existen y existirán, deben ser evaluados y estudiados, con el fin de determinar qué factores son los que afectan su rendimiento y productividad, y posteriormente verificar su importancia y aporte (Villanueva Joaquin \& Bustos Tirado, 2020).

Para un ingeniero civil $o$ cualquier profesional que se encuentre dentro de la industria de la construcción, es importante evaluar estos rendimientos en cada uno de sus proyectos y de las actividades, con el fin de poder desarrollar la capacidad para la toma de mejores decisiones que lleven a la solución más optima según la problemática presentada, las herramientas disponibles y el contexto en que se encuentre (Riaño Anaya, 2021)"type":"articlejournal"\},"uris":[“http://www.mendeley.com/ documents/?uuid=57c2f096-49f2-4c56-a8d8-d1 6365dc7440"]\}],"mendeley":\{“formattedCitation": "(Riaño Anaya, 2021.

Es por esta razón, que este trabajo de investigación pretende determinar la diferencia en los rendimientos calculados mediante el sistema constructivo industrializado para las actividades de levantamiento de muros de carga y construcción de placas de entre piso. Con los resultados obtenidos podremos determinar cuales son los aspectos más relevantes en cada elemento estructural, sin embargo, al tratarse de una misma metodología, se espera que no existan grandes diferencias.

\section{METODOLOGÍA}

La metodología utilizada abarcó el seguimientoconstructivodeestos procedimientos en 5 viviendas diferentes, en un proyecto de viviendas de interés social dentro de la ciudad de Cúcuta. Mediante la implementación de un formato de recolección de datos se promedió los diferentes materiales y tiempos de ejecución en estas dos actividades. En primera instancia se realiza el cálculo de rendimientos en formaletas para sistema industrializado en la actividad de levantamiento de muros y construcción de placa entre piso, posteriormente se analizarán de manera comparativa los resultados obtenidos.

\section{DESARROLLO}

\section{Cálculo de rendimientos en formaletas para sistema industrializado en la actividad de levantamiento de muros y construcción de placa entre piso}

Para el cálculo de rendimientos, se realizó un seguimiento de obra detallado a este proyecto de vivienda de interés social, donde se 
intervenía la construcción de muros de carga y placa maciza de entre piso, debido a que estos elementos estructurales se construyen confinados en formaletas de acero, para su posterior vaciado de concreto.

Estas viviendas fueron construidas en sistemas bifásicos, donde primero consistía en realizar el levantamiento en muros fundidos en concreto y posteriormente se procede a construir la placa maciza de entrepiso, por lo tanto, se pudieron tomar los datos de manera independiente en cada una de estas fases de construcción.

Debido a que se tomaron datos de las 5 viviendas, en la tabla 1 se logra apreciar el promedio encontrado en cada una de las actividades, estos datos vienen de un formato establecido que presenta cada una de las sub actividades que conforman estos procedimientos, sub actividades que afectan de manera directa con el tiempo de utilización de la formaleta, dentro de las cuales se incluye, el tiempo de armado, colocación del acero (malla electrosoldada para estos casos), vaciado del concreto, fraguado y desencofrado. Sub actividades que se aplican para la ejecución tanto de la placa como de los muros, especificando su duración obtenida y el gasto en su unidad de medida.

\begin{tabular}{|c|c|c|c|}
\hline \multirow{2}{*}{ SUB ACTIVIDAD } & \multirow{2}{*}{ UNIDAD } & \multicolumn{2}{|c|}{ PROMEDIO } \\
\hline & & MUROS & PLACAS \\
\hline Cuadrilla & $\begin{array}{l}\text { Oficial + } \\
\text { Ayudantes }\end{array}$ & 12 & 9 \\
\hline Cuadrilla Menor & $\begin{array}{l}\text { Oficial + } \\
\text { Ayudante }\end{array}$ & 2 & 2 \\
\hline $\begin{array}{l}\text { Especificaciones del } \\
\text { concreto }\end{array}$ & & 3000 psi & $3000 \mathrm{psi}$ \\
\hline Cantidad de concreto & M3 & 10 & 7,2 \\
\hline Tipo de Formaleta & & metálica & metálica \\
\hline $\begin{array}{l}\text { Cantidad de } \\
\text { formaleta }\end{array}$ & UND & 112 & 60 \\
\hline Cantidad de Parales & UND (3m) & 134 & 142 \\
\hline Cantidad de Cerchas & UND (3m) & 21 & 20 \\
\hline Cantidad de Cerchas & UND (2m) & 21 & 20 \\
\hline Área & M2 & 105 & 32,46 \\
\hline Volumen de Placa & M3 & 8,26 & 4,0 \\
\hline Armado de Formaleta & $\mathrm{H}$ & 4,2 & 3,4 \\
\hline $\begin{array}{l}\text { Armado malla } \\
\text { electrosoldada }\end{array}$ & $\mathrm{H}$ & 8,2 & 2,5 \\
\hline $\begin{array}{l}\text { Kg de Malla } \\
\text { empleados }\end{array}$ & $\mathrm{H}$ & 324,5 & 120,5 \\
\hline $\begin{array}{l}\text { Tiempo total de } \\
\text { Vaciado }\end{array}$ & $\mathrm{H}$ & 2,12 & 0,12 \\
\hline Codal & $\mathrm{H}$ & 0 & 0,20 \\
\hline Fraguado & $\mathrm{H}$ & 11,5 & 13,8 \\
\hline Desencofrado & $\mathrm{H}$ & 4,2 & 3,0 \\
\hline Curado & $\mathrm{H}$ & 2,5 & 0,70 \\
\hline
\end{tabular}

Tabla1. Promedios sub actividades 
Por lo tanto, tenemos que el rendimiento es la cantidad de medida realizada de una actividad con respecto a la composición de la cuadrilla, expresada en unidades de hora/ hombre (Hh) (Rojas Torres, 2020)"type":"articlejournal"],"uris":[“http://www.mendeley.com/ documents/?uuid=ca5e4d5f-6c71-4a01-a88a-df d26b600694"]\}],"mendeley":\{“formattedCitation": "(Rojas Torres, 2020

Obteniendo así la ecuación 1.

$$
\begin{gathered}
\text { Rendimiento }=\frac{\text { Cantidad de personas } x \text { Duracion de actividad }}{\text { Cantidad de obra ejecutada }} \\
\text { Rendimiento }=\frac{\text { Hora Hombre }}{\text { Unidad }}
\end{gathered}
$$

A continuación, se explica detalladamente un ejemplo de cómo se realiza este cálculo, y este procedimiento se replicará con cada actividad.

$$
\begin{aligned}
& \text { Rendimiento Formaleta Muros }=\frac{12 \text { hombres } x 4,2 \mathrm{H}}{105 \mathrm{~m}^{2}} \\
& \text { Rendimiento Formaleta Muros }=0,4800 \frac{\mathrm{Hh}}{\mathrm{m}^{2}}
\end{aligned}
$$

En la tabla 2 se encuentran los resultados del este cálculo para cada una de las sub actividades en los dos procedimientos.

\begin{tabular}{ccc}
\hline ACTIVIDAD & MUROS & PLACAS \\
Armado de & 0,4800 & 0,94269 \\
formaleta & & \\
Acero & 0,0505 & 0,04149 \\
Vaciado & 3,0799 & 0,27000 \\
Desencofrado & 0,4800 & 0,83179 \\
Curado & 0,0476 & 0,04313 \\
\hline
\end{tabular}

Tabla 2. Rendimientos de actividades

\section{ANÁLISIS COMPARATIVO DE LOS RESULTADOS}

Esta figura representa los datos de la tabla 2, donde se puede evidenciar cuales actividades son las que demandan mayor consumo de equipo y materiales, , observando que existen algunas actividades que varían de manera considerablemente como lo es el caso del vaciado de concreto.

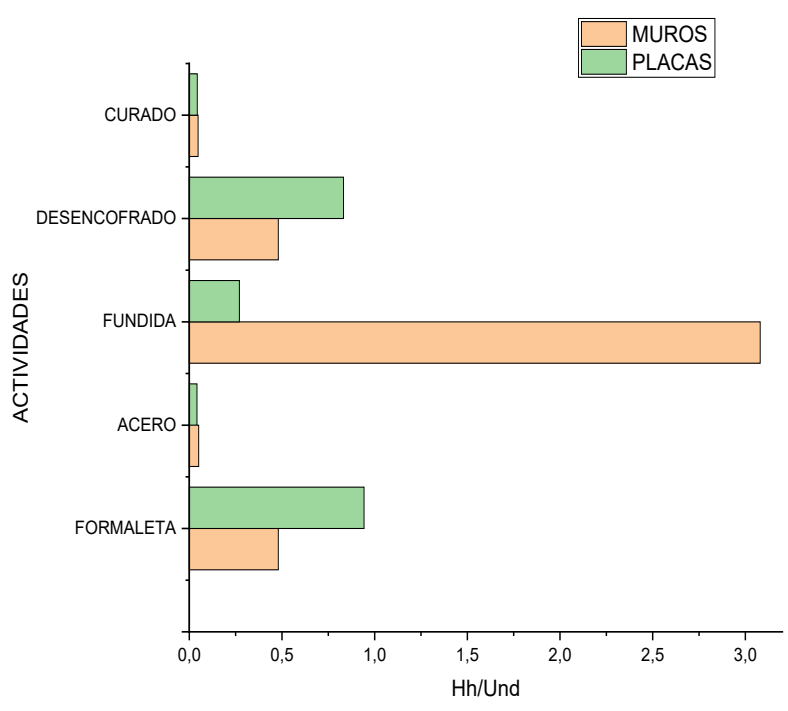

Figura 1. Comparación de los rendimientos.

\section{CONCLUSIONES}

El resultado demuestra que existe una ventaja en la utilización de este tipo de formaletas con respecto a la construcción de placas de entrepiso, esta ventaja, seguramente se debe a la distribución de estas formaletas de manera horizontal que necesitan crear lo que se considera como diafragma rígido.

Como aspectos generales se puede determinar que el armado de formaleta en placas de entrepiso es el doble de tardía con respecto a el armado de formaleta de los muros de carga. La creación de la malla electrosoldada y su instalación es un $21 \%$ más eficiente en las placas de entre piso, así mismo el vaciado del concreto en muros tiene un rendimiento 11 veces inferior que la de la placa. El desencofrado de los muros de carga es un $57 \%$ más rápido que el desencofrado de la placa entre piso y por último, tenemos que el curado del concreto no varía más o menos $10 \%$ respecto uno del otro. 
En el caso de los muros, el armado de estas formaletas quizás es un poco más complicado, debido a que cada muro independiente debe tener su formaleta autónoma.

Así mismo, se determina que un factor importante a la hora de fundir los concretos es la geometría y la posición en que se encuentran estos, puesto que, en un muro puede existir una mala segregación, es por esto que se requiere el uso de herramientas como martillos de goma y vibradores con el fin de garantizar la correcta colocación de cada uno de los materiales del concreto, el uso de estas herramientas conlleva a un mayor esfuerzo y por lo tanto a un mayor tiempo con respecto a una losa de entre piso, ya que la altura en este segundo elemento estructural, facilita el procedimiento de la colocación y distribución del concreto.

Con respecto al desencofrado, debido a que los muros soportan la carga de la placa, existe menos riesgo de deformación del mismo, sin embargo, la placa de entrepiso, puede presentar problemas de deflexión o incluso punzonamiento si estos elementos portantes no se retiran de la manera más adecuada.

\section{BIBLIOGRAFÍA}

Aguirre Almache, R. C., \& Zúñiga Chimbo, A. V. (2019). Análisis comparativo de los sistemas constructivos-aporticado y manoportable empleados en la construcción de conjuntos habitacionales. Quito: UCE.

Espinoza, A. S., \& MorÃ, J. V. (2017). Sistemas constructivos ventajas y desventajas. Observatorio de La Economía Latinoamericana, 234.

Flores, L. E. L. (2018). Análisis y evolución de los sistemas constructivos prefabricados, impacto ambiental e interacción con el sistema constructivo tradicional mexicano= Analysis and evolution of prefabricated building systems, environmental impact and interaction with the traditional Mexican construction system. Anales de Edificación, 4(3), 44-51.

Mosquera Ayala, L., \& Noreña Trejos, M. A. (2021). Estudio de viabilidad ambiental y financiero sobre las técnicas de la construcción sostenible que pueden adoptarse en la construcción tradicional de viviendas en Colombia.

Orozco Herrera, D. C., \& López Toro, A. (2020). Déficit habitacional y pobreza: un abordaje desde las políticas públicas de vivienda en Colombia. Caso Cien mil viviendas gratis para los colombianos y Tarso mi techo solidario (2012-2019)(dossier).

Puerto Castro, O. M. (2015). Informe de pasantía en el proyecto de vivienda centro residencial Avatar. Universidad Santo Tomas.

Riaño Anaya, L. X. (2021). Manejo financiero de una empresa de servicios dedicada al alquiler de equipos para construcción ubicada en la ciudad de Duitama-Boyacá.

Rojas Torres, L. K. (2020). Análisis de rendimientos de equipos en el proceso de perforaciones de anclajes para taludes según el tipo de suelo e imprevistos.

Tovar, A. (2021). Construcción industrializada, el cambio necesario para los nuevos desafíos. Cemento Hormigón, 1003, 3.

Villanueva Joaquin, L. E., \& Bustos Tirado, J. O. (2020). Optimización de los procesos productivos utilizando Value Stream Mapping (VSM) en los procesos constructivos de placa de ascensor, placa de escalera y losa maciza "sector 4": Caso: Proyecto "Condominio Bolivia $n^{\circ} 848$ " Breña-Lima. 